Check for updates

Cite this: RSC Chem. Biol., 2020, 1,98

Received 25th April 2020, Accepted 20th May 2020 DOI: $10.1039 / \mathrm{d} 0 \mathrm{cb} 00052 \mathrm{c}$ rsc.li/rsc-chembio

\section{Tools for functional dissection of site-specific O-GlcNAcylation}

\begin{abstract}
Andrii Gorelik (D) $\nmid \ddagger^{a}$ and Daan M. F. van Aalten (i) $\neq^{*^{a b}}$
Protein O-GlcNAcylation is an abundant post-translational modification of intracellular proteins with the monosaccharide $N$-acetylglucosamine covalently tethered to serines and threonines. Modification of proteins with O-GlcNAc is required for metazoan embryo development and maintains cellular homeostasis through effects on transcription, signalling and stress response. While disruption of O-GlcNAc homeostasis can have detrimental impact on cell physiology and cause various diseases, little is known about the functions of individual O-GlcNAc sites. Most of the sites are modified substoichiometrically which is a major challenge to the dissection of O-GlcNAc function. Here, we discuss the application, advantages and limitations of the currently available tools and technologies utilised to dissect the function of O-GlcNAc on individual proteins and sites in vitro and in vivo. Additionally, we provide a perspective on future developments required to decipher the protein- and site-specific roles of this essential sugar modification.
\end{abstract}

\section{Post(co)-translational protein O-GlcNAcylation}

\footnotetext{
${ }^{a}$ Centre for Gene Regulation and Expression, School of Life Sciences, University of Dundee, Dundee, UK. E-mail: dmfvanaalten@dundee.ac.uk

${ }^{b}$ Institute for Molecular Precision Medicine, Xiangya Hospital, Central South University, Changsha, China

$\dagger$ Current address: Department of Chemistry, Imperial College London, London, UK; The Francis Crick Institute, London, UK.

\$ AG and DvA wrote the manuscript.
}

Post-translational modifications (PTMs) expand the function and regulation of proteins beyond the genetically encoded polypeptide. The number of known types of PTMs is close to several hundred, ${ }^{1}$ ranging from substantial alterations (proteolytic cleavage resulting in large protein fragments, attachment of ubiquitin chains, glycosylation with polysaccharides) ${ }^{2-4}$

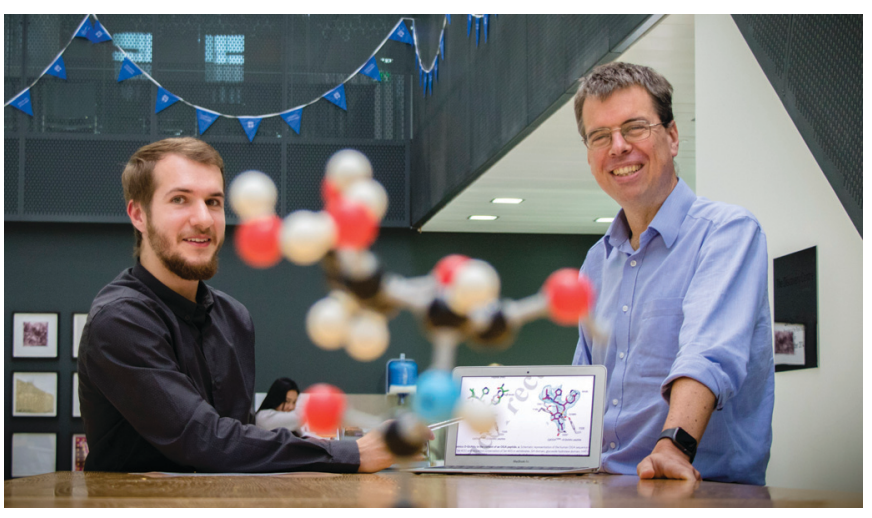

Andrii Gorelik and Daan van Aalten

Andrii Gorelik (left in the picture) received a Bachelor's degree in industrial biotechnology in 2014 from the Kyiv Polytechnic Institute (Ukraine). He then moved to the School of Life Sciences at the University of Dundee (UK) to complete a Wellcome Trust-funded PhD in 2018, establishing novel methods for functional dissection of site-specific protein O-GlcNAcylation under the supervision of Prof. Daan van Aalten. During his time in Dundee, he developed a particular interest in post-translational modifications (PTMs). He is presently undertaking postdoctoral research at the Francis Crick Institute and Imperial College London with Prof. Ed Tate in the field of PTM chemical biology, focusing on protein N-myristoylation.

Daan van Aalten (right in the picture) is visiting professor at Xiangya Hospital, Central South University, Changsha, China and Professor of Biological Chemistry at the School of Life Sciences, Dundee. Originally graduated as a chemist (1994) followed by a biocomputing PhD (1997), Daan has been working on the interface between cell signalling and glycobiology since joining Dundee as a PI (1999) using a multidisciplinary approach covering everything from synthetic chemistry to genetics. Daan's work has been focused in the O-GlcNAc signalling field for the past 10 years where he has developed chemical biology tools, and uncovered novel molecular/biological mechanisms of this modification. 
a
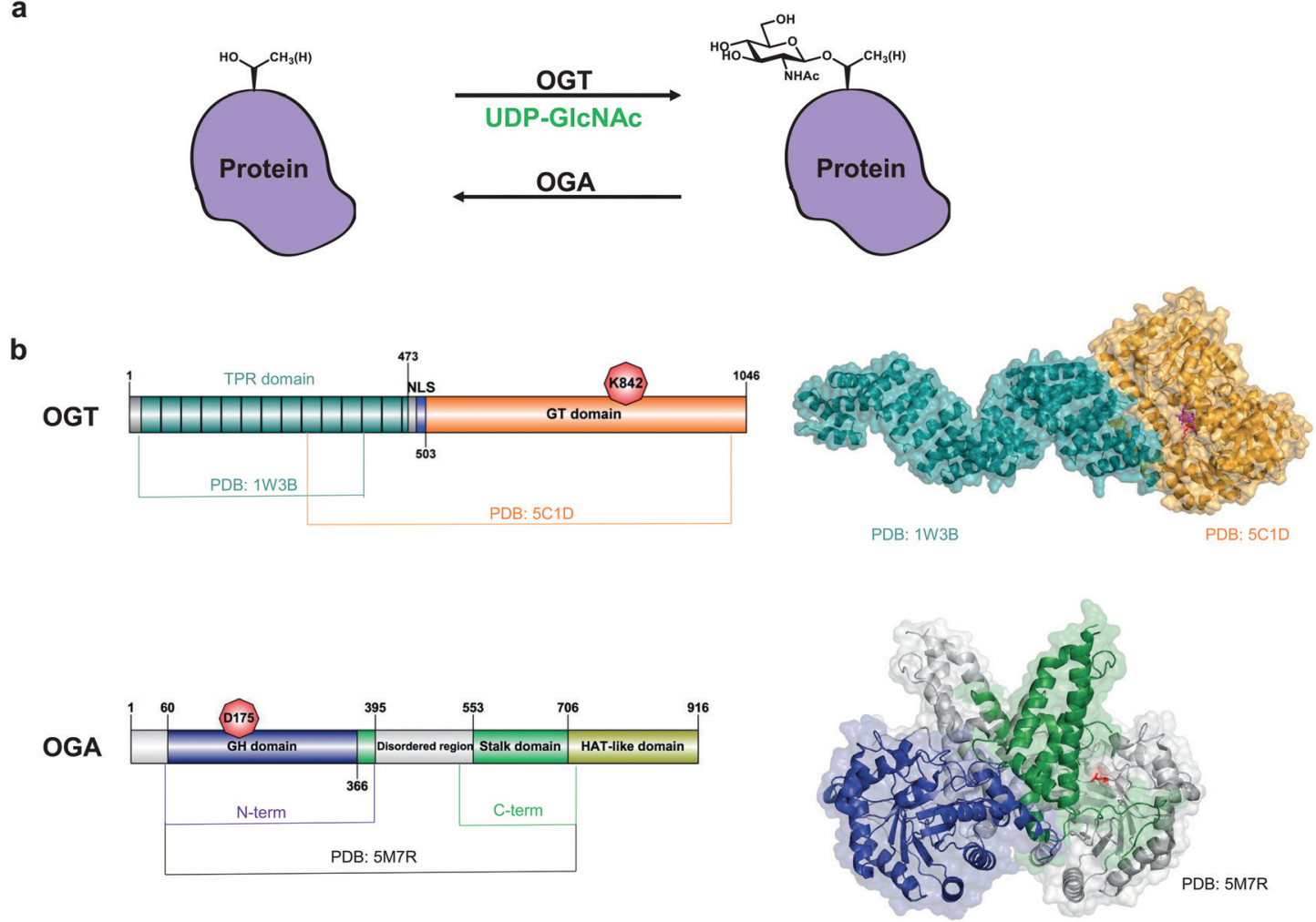

Fig. 1 O-GlcNAc cycling on nucleocytoplasmic proteins. (a) OGT and OGA control the addition and removal of GlcNAc on serine and threonine residues. (b) Domain architecture and structures of OGT (composite of PDB: 1W3B and PDB: 5C1D) and OGA (PDB: 5M7R, lacks the disordered region). Catalytic site residues K842 (OGT) and D175 (OGA) are coloured in red. GT domain - glycosyltransferase domain. NLS - nuclear localization sequence, ${ }^{40}$ $\mathrm{GH}$ domain - glycoside hydrolase domain.

to small tags (phosphorylation, acetylation, methylation, lipidation). ${ }^{5-8}$

One of these PTMs, O-GlcNAcylation, is the attachment of a single O-linked $\mathrm{N}$-acetylglucosamine onto serine and threonine side chains of intracellular proteins, ${ }^{9}$ which, in some cases, is also thought to be co-translational. ${ }^{10}$ Installation of the sugar is mediated by the O-GlcNAc transferase (OGT) $)^{11,12}$ on nuclear and cytoplasmic proteins, covering $10-20 \%$ of the whole human proteome (Fig. 1a). ${ }^{13}$ The O-GlcNAcase (OGA) opposes OGT by hydrolysing the O-glycosydic bond to release GlcNAc. ${ }^{14}$ Thus, a single pair of enzymes makes O-GlcNAc a reversible and highly dynamic modification that has been implicated in a myriad of processes such as signalling, metabolism, stress response and transcription. ${ }^{15}$

OGT is a family GT41 GT-B glycosyltransferase ${ }^{16}$ that consists of a 13.5 tetratricopeptide repeat (TPR) domain and a Rossman-like catalytic domain (Fig. 1b). ${ }^{17}$ Although the 13.5-TPR OGT is the major isoform, two additional isoforms may arise from alternative splicing and alternative start codons: a truncated OGT with 2.5 TPRs localised in the nucleus and cytoplasm and a 9.5-TPR OGT containing a mitochondrial targeting sequence. ${ }^{18,19}$ The function of the latter two isoforms is unclear. Moreover, it appears that full-length nucleocytoplasmic OGT is sufficient for O-GlcNAcylation of mitochondrial proteins. ${ }^{20} \mathrm{O}-$ GlcNAc transfer by OGT involves an ordered bi-bi catalytic mechanism: binding of the donor sugar nucleotide
UDP-GlcNAc is followed by the target protein acceptor substrate. ${ }^{17,21}$ A second catalytic activity of OGT, host cell factor-1 (HCF-1) cleavage, is performed in the same active site and results in proteolytic maturation of this transcriptional co-regulator. ${ }^{22}$ More recently, OGT was shown to possess a third (and unexpected) activity - catalysis of S-GlcNAc transfer onto cysteines. $^{23,24}$ Although the crystal structures of the TPRs and the truncated OGT have been solved (Fig. 1b), ${ }^{17,25}$ the full-length OGT structure is yet to be determined and may provide a further understanding of how OGT recognizes its substrates. The TPR domain of OGT mediates substrate recognition through a so-called asparagine ladder. ${ }^{17}$ Using protein microarrays and mutational analysis, the five asparagines in the lumen of the TPR domain have been shown to contribute to the binding of OGT protein substrates beyond its active site. $^{26,27}$ Recently, additional aspartates in the TPR domain that influence recognition of some OGT substrates have been identified. $^{28}$ OGT does not utilise a defined motif for O-GlcNAcylation. However, in a high-throughput screen a preferred O-GlcNAc sequon has been determined as T-P-V-gS/T-R$\mathrm{A}^{12}$ which is in good agreement with P-X-gT-X-A and P-V-g $\mathbf{S}^{29}$ and $\mathrm{P} / \mathrm{V}-\mathrm{P} / \mathrm{V}-\mathrm{V}-\mathrm{gS} / \mathrm{T}-\mathrm{S} / \mathrm{T},{ }^{30,31}$ previously identified in large-scale proteomics studies.

OGA is a GH84 family glycoside hydrolase ${ }^{16}$ consisting of an $\mathrm{N}$-terminal catalytic domain and a histone acetyltransferase (HAT)-like domain separated by a disordered region and a stalk 
domain (Fig. 1b). The HAT-like domain lacks the ability to bind acetyl coenzyme A (the substrate for acetylation) and its role remains enigmatic. ${ }^{32}$ The putative short human OGA isoform lacks the HAT-like domain and a portion of the stalk domain, resulting in reduced activity due to the inability to form a functional dimer. ${ }^{33}$ Overexpression of this isoform with a GFP tag results in its localisation to lipid droplets. ${ }^{34}$ However, the short OGA isoform has not been detected at an endogenous protein level. The first crystal structures of a monomeric bacterial orthologue of human OGA from Clostridium perfringens (CpOGA) in complex with its substrate peptides exhibited a V-shaped peptide conformation in the active site. ${ }^{35,36}$ Later it was shown that human OGA forms a dimer with a substrate-binding cleft where O-GlcNAc peptides assume the same V-shaped conformation upon binding in the active site, confirming the findings obtained with CpOGA. ${ }^{33,37,38}$ While the interactions of OGA active site residues with GlcNAc are conserved, exactly how (and whether) protein substrates bind beyond the active site is unknown. ${ }^{39}$ OGA itself contains a single O-GlcNAcylation site at Ser405, which may regulate OGA stability. ${ }^{24}$

The roles of O-GlcNAc on proteins have been studied in animal models (such as mice and fruit flies), where OGT deletion is lethal and causes severe developmental abnormalities. ${ }^{41-43}$ Excess of O-GlcNAc can also have negative impact on normal cell physiology as OGA knockout is lethal in mice, ${ }^{44}$ whereas increased O-GlcNAc levels have been linked to oncogenic reprogramming. ${ }^{45}$ Since its discovery, the relationship between O-GlcNAcylation and phosphorylation has been closely studied due to the frequent occurrence on the same or adjacent Ser/Thr residues which results in mutual regulation. ${ }^{46}$ The role of O-GlcNAc in stress response is exemplified by its participation in stress granule formation and oxidative stress. ${ }^{47}$ At individual protein level, lack of O-GlcNAc on tau and $\alpha$-synuclein has been associated with pathological brain conditions such as Alzheimer's and Parkinson's disease.$^{48,49}$ Moreover, O-GlcNAc can mediate transcription through modification of RNA Polymerase $\mathrm{II}^{50}$ and regulate autophagosome maturation by modifying SNAP29 depending on the cellular metabolic state. ${ }^{51}$

Transcriptionally, OGT expression can be decreased through nuclear degradation of an intron-retained OGT transcript in response to high overall O-GlcNAcylation levels to maintain O-GlcNAc homeostasis. ${ }^{52}$ Similar to OGT, OGA expression is also responsive to the modulation of overall O-GlcNAc modification. Pharmacological inhibition of OGA in cells increases total O-GlcNAcylation levels, while also increasing OGA expression and decreasing expression of OGT as a compensatory mechanism. ${ }^{53}$

Recently, ogt missense mutations associated with X-linked intellectual disability have been identified. ${ }^{54}$ These occur in the TPR domain of OGT ${ }^{54-56}$ as well as in the catalytic domain. ${ }^{57,58}$ Some of these mutations cause a decrease in endogenous OGT activity. ${ }^{55,57}$ Therefore, it is possible that abolished O-GlcNAcylation status on a subset of OGT substrates or even a single substrate/site could contribute to the intellectual disability phenotype, an avenue that is currently being explored in our laboratory using several methods discussed in this review.
Currently, the O-GlcNAc field is experiencing rapid growth with researchers from related disciplines discovering that O-GlcNAc modulates the function of their proteins of interest. Examples include discoveries of the role of O-GlcNAcylation in preventing the aggregation of a Polycomb member, polyhomeotic, in Drosophila, ${ }^{59}$ as well as in controlling the anti-inflammatory function on receptor-interacting serine/threonine-protein kinase 3 $(\text { RIPK3 })^{60}$ and in activating phosphoglycerate kinase 1 (PGK1) to promote glycolysis, ${ }^{61}$ to name a few. As the roles of O-GlcNAc are becoming recognized, there is an emerging need for tools to dissect site-specific O-GlcNAcylation.

\section{Visualisation of protein- and site-specific O-GIcNAcylation}

A crucial step in studying any PTM is its detection. Although mass spectrometry (MS) provides a high-throughput method for O-GlcNAc identification and site-mapping (reviewed elsewhere ${ }^{62}$ ), it is complicated by time-consuming sample preparation, expensive equipment and (often difficult) analysis. Therefore, the use of gel and western blot methods is generally required which will be discussed below.

In the early days of the field, O-GlcNAc was detected by galactosyltransferase labelling with an $\left[{ }^{3} \mathrm{H}\right]$-galactose followed by autoradiography and by lectin binding (for instance, with fluorescently labelled wheat germ agglutinin, which binds terminal GlcNAc residues). ${ }^{63,64}$ This was later surpassed by a superior chemoenzymatic method based on the mutant $\beta-1,4$ galactosyltransferase $\left(\mathrm{GalT}^{\mathrm{Y} 289 \mathrm{~L}}\right)$ that transfers an azide derivative of GalNAc (GalNAz) onto O-GlcNAcylated proteins. ${ }^{65}$ The azide provides a reactive handle for the attachment of tags using copper-catalysed azide-alkyne cycloaddition (CuAAC) or copper-free strain-promoted azide-alkyne cycloaddition (SPAAC), ${ }^{66,67}$ allowing qualitative and quantitative detection of O-GlcNAc. ${ }^{66,68}$ The use of mass tags (such as polyethylene glycols PEG5000- or PEG2000-alkyne) allows resolution of GlcNAcmodified proteins on a gel and determination of absolute stoichiometry using a western blot with an antibody against the target protein (eliminating the need for pulldowns and O-GlcNAcspecific antibodies). ${ }^{66}$ Additionally, the number of bands shifted indicates the number of O-GlcNAc sites. Unfortunately, the detection limit of the PEGylation method is approximately 5\% (depending on the quality of the antibody) and the labelling efficiency relies on full completion of the galactosyltransferase reaction. ${ }^{66}$ The efficiency of the subsequent chemical addition of a PEG mass tag may also vary depending on the reagent concentration. To address this issue, the SPAAC reaction has been optimised using a synthetic protein standard (bearing stoichiometric O-GlcNAc modification) and commercial reagents. ${ }^{69}$ As an alternative to mass tagging, a native polyacrylamide gel electrophoresis (native PAGE) method based on co-polymerised CpOGA has been developed in our laboratory (C. Fu and D. M. F. van Aalten, Analyst, in press). The retardation of an O-GlcNAcmodified band happens as a result of high-affinity binding by the catalytically inactive $C p$ OGA. Conveniently, this approach does not require any chemical modification of the sample.

Sometimes the stoichiometry of O-GlcNAc on a given protein is below the detection limit of antibodies. In order to amplify 
the O-GlcNAc signal in such cases, a proximity-ligation based method has been developed. First, O-GlcNAc is chemoenzymatically labelled with GalNAz, followed by a reaction with an alkyne-biotin. The next step is the coupling of antibody-DNA conjugates targeted to either biotin or the protein of interest. If the protein is O-GlcNAcylated, the antibodies are brought into proximity, leading to ligation of DNA tags. qPCR is subsequently performed to quantify the signal. ${ }^{70}$ While this method allows detection of O-GlcNAcylated proteins in small amounts of sample, the specificity of O-GlcNAc signal heavily relies on the specificity of an antibody against a protein of interest which could otherwise detect off-targets.

The donor substrate promiscuity of OGT has been extensively exploited to generate various metabolic reporters for global O-GlcNAc profiling by mass-spectrometry and in-gel visualisation. These modified sugars can be fed to cells where they enter specific metabolic pathways and can be utilised by OGT as donor substrates. These include GlcNAc derivatives with alkyne and azide functionalities such as GlcNAz, ${ }^{71}$ GlcNAlk, ${ }^{72}$ 6AzGlcNAc, ${ }^{73}$ 4-deoxy-GlcNAz, ${ }^{74}$ 2AzGlc, ${ }^{75}$ 6AlkGlcNAc, ${ }^{76}$ $6 \mathrm{AzGlc}^{77}$ and GlcNDAz. ${ }^{78}$

Antibodies remain the most popular tool to detect O-GlcNAc. The two most widely used pan-specific O-GlcNAc antibodies are RL2 (originally developed for detecting nuclear pore proteins) ${ }^{64,79}$ and CTD110.6 (developed based on the O-GlcNAcylated RNA polymerase II C-terminal domain). ${ }^{80}$ Although both of these commercially available antibodies are monoclonal, RL2 exclusively binds to O-GlcNAc, while CTD110.6 may also recognise $N$-GlcNAc, GlcNAcylated O-mannose, cross-reacts with terminal $\beta$-GlcNAc on complex $N$-glycans of cell surface glycoproteins and more recently has been shown to efficiently detect S-GlcNAc on cysteines. ${ }^{24,81-84}$ Therefore, care must be taken when using CTD110.6 antibody whose specificity must be tested in each case with an OGA-treated negative control. Although RL2 binds O-GlcNAc with greater specificity, it may not recognise O-GlcNAcylation in certain contexts such as demonstrated with $\alpha$-synuclein stoichiometrically O-GlcNAcmodified at several reported O-GlcNAcylation sites. ${ }^{85}$

Unlike in the field of phosphorylation, very few site-specific O-GlcNAc antibodies have been generated. Nevertheless, these have greatly aided the study of site-specific O-GlcNAc modification and are summarised in Table 1. For instance, reciprocal interplay between O-GlcNAcylation and proximal phosphorylation could be examined using antibodies against O-GlcNAcylated Thr58 on c-Myc (first site-specific O-GlcNAc antibody ever produced) ${ }^{86}$ and O-GlcNAcylated Ser400 on tau. ${ }^{46}$ Our laboratory had generated a Ser395-O-GlcNAc-specific TGF- $\beta$ activated kinase-1 (TAK1) binding protein-1 (TAB1) antibody that revealed increased O-GlcNAcylation as a modulator of TAK1 signalling upon IL-1 stimulation and osmotic stress. ${ }^{87}$ More recently, we produced a Ser517-O-GlcNAc-specific CRMP2 antibody to show an age-dependent increase in CRMP2 O-GlcNAcylation associated with short-term memory impairment. ${ }^{88}$

\section{Enzymatic methods for generating protein-specific O-GlcNAcylation}

Access to stoichiometrically modified proteins is often required to dissect the mechanistic consequences of PTMs in vitro and in vivo. However, producing highly O-GlcNAc-modified proteins is challenging. Traditionally, the simplest approach to obtain an O-GlcNAcylated protein of interest has been in vitro reaction with OGT (Fig. 2a). ${ }^{96}$ Despite the ease of implementation, to achieve high O-GlcNAc stoichiometry, long incubation times are generally needed which in itself may have undesired effects on the stability of the protein under investigation. Alternatively, to preserve protein stability, OGT can be co-expressed with its substrates in E. coli or insect cells (Fig. 2b). Application of this system in a bacterial setting has been demonstrated for the RNA-Polymerase II C-terminal domain, TAB1, calcium/ calmodulin-dependent protein kinase type IV (CaMKIV), Tau, Coactivator Associated Arginine Methyltransferase 1 (CARM1) and nuclear pore glycoprotein p62 (nup62). ${ }^{96-98}$ However, an endogenous glycosidase in $E$. coli may compromise the yield of the O-GlcNAc-modified proteins produced with this approach. ${ }^{99}$ In insect (Sf9) cells simultaneous expression of CREB and OGT led to a three-fold increase of CREB glycosylation to almost $90 \% .{ }^{66}$ Although this co-expression approach may not be compatible with all OGT substrates, the main advantage is the ability to produce co-translationally O-GlcNAc-modified proteins which is impossible via OGT reaction in vitro.

Recombinant O-GlcNAc-modified protein on its own is usually not sufficient to dissect the function of this modification.

Table 1 Site-specific O-GlcNAc antibodies generated to date

\begin{tabular}{|c|c|c|}
\hline O-GlcNAc antibody & Site (human) & Ref. \\
\hline c-Myc & Thr58 & 86 \\
\hline Collapsin response mediator protein-2 (CRMP2) & Ser517 & 88 \\
\hline Histone $\mathrm{H} 2 \mathrm{~A}$ & Ser40 & 89 \\
\hline Histone $\mathrm{H} 2 \mathrm{~A}$ & Thr101 & www.glycoscientific.com/ \\
\hline Histone $\mathrm{H} 2 \mathrm{~B}$ & Ser112 & 90 \\
\hline Histone $\mathrm{H} 3$ & Thr32 & www.glycoscientific.com/ \\
\hline Histone H4 & Ser47 & www.glycoscientific.com/ \\
\hline Insulin receptor substrate-1 (IRS1) & Ser1011 & 91 \\
\hline Insulin receptor substrate-2 (IRS2) & Thr1155 & www.glycoscientific.com/ \\
\hline NAD-dependent protein deacetylase Sirtuin-1 (SIRT1) & Ser549 & 92 \\
\hline Tau & Ser400 & 93 and 94 \\
\hline TGF-beta-activated kinase 1-binding protein 1 (TAB1) & Ser395 & 87 \\
\hline TGF-beta-activated kinase 1-binding protein 3 (TAB3) & Ser408 & 95 \\
\hline
\end{tabular}




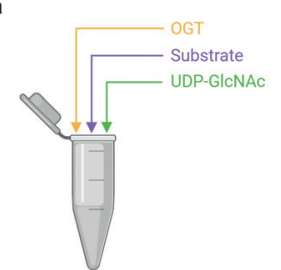

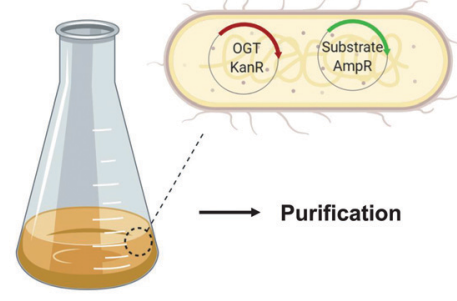

c

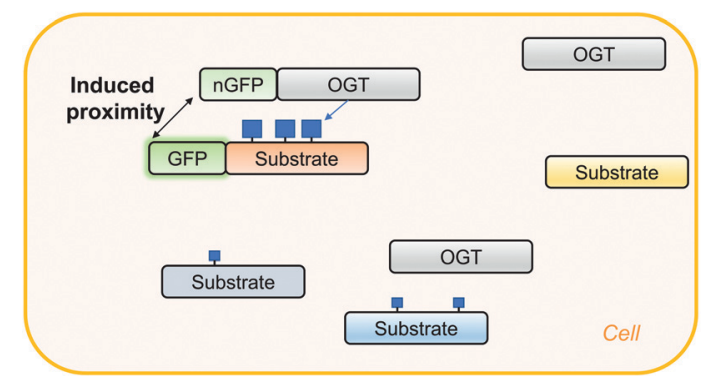

Fig. 2 Methods for generating protein-specific O-GlcNAcylation. (a) In vitro OGT reaction. (b) Co-expression of OGT with its substrate in E. coli, followed by purification. KanR - kanamycin resistance, AmpR ampicillin resistance. (c) Proximity OGT approach for protein-selective O-GlcNAcylation in cells. ${ }^{100}$ nGFP - anti-GFP nanobody. Blue squares denote O-GIcNAc

To acquire control over a single-protein O-GlcNAcylation status in a complex cellular environment, a creative way to induce proximity of OGT to its substrates in cells has been proposed recently (Fig. 2c). ${ }^{100}$ Exploiting the fusion of OGT to a nanobody against a tag (GFP or RFP) on a protein of interest or a nanobody against endogenous protein target, Woo and colleagues managed to increase O-GlcNAcylation stoichiometry on a subset of OGT substrates. In its current form this proximity induction relies on the overexpression of OGT which concomitantly elevates global O-GlcNAc levels. This strategy may have potential to increase O-GlcNAcylation on a protein of interest, if the impact of OGT overexpression on the global O-GlcNAcome can be addressed. Alternatively, other proximity approaches could be explored, such as the use of aptamers or small molecules (PROTAC principle). For example, the use of a heterobifunctional small molecule has already been utilised to bring protein phosphatase 1 (PP1) in proximity to its targets

resulting in a protein-specific decrease in phosphorylation of AKT kinase and EGFR. ${ }^{101}$ One of the most important considerations for the future development of such probes is that the interaction of the proximity-inducing molecules does not affect the catalytic or scaffolding function of OGT or its substrate.

Current methods to increase protein-specific O-GlcNAcylation still result in heterogeneous mixtures of modified and unmodified proteins and make functional analysis particularly cumbersome in case of multiple glycosylation sites. Thus, knowledge of specific O-GlcNAc sites and application of approaches to generate sitespecifically O-GlcNAcylated proteins could circumvent this issue.

\section{Chemical biology methods for dissecting site-specific O-GlcNAcylation in vitro}

One approach that has been explored to incorporate sitespecific O-GlcNAcylation is expressed protein ligation (EPL), a semi-synthetic strategy to obtain homogenous and stoichiometric site-specific PTMs of a target protein (Fig. 3a). ${ }^{102,103}$ EPL involves synthesis of a peptide bearing a desired functionality (e.g. post-translational modification), which is then ligated via S-to- $N$ acyl-transfer to a thioester generated from a recombinant intein-fusion protein. ${ }^{102}$ To date, tau, ${ }^{104} \alpha$-synuclein, ${ }^{49,85,105}$ HSP $27^{106}$ and an unnaturally modified ubiquitin ${ }^{69}$ are the only four reported O-GlcNAcylated proteins produced using EPL. This method has been used to dissect the mechanistic consequences of O-GlcNAcylation on $\alpha$-synuclein (Thr72, Thr75, Thr81, Ser87), ${ }^{49,85,105}$ which is a small $(<15 \mathrm{kDa})$ protein amenable to EPL. This approach revealed that O-GlcNAc on $\alpha$-synuclein affects aggregation and toxicity in vitro. ${ }^{85}$ Apart from a handful of O-GlcNAcylated proteins, S-GlcNAcylated casein kinase $2(\mathrm{CK} 2)^{107}$ and $\alpha$-synuclein ${ }^{108}$ have been produced by EPL in order to increase the stability of the modification against hydrolysis. Through structural and biophysical studies it was shown that S-GlcNAc serves as a good non-hydrolysable analogue for O-GlcNAc that can be recognized by some O-GlcNAc-specific antibodies (Ser395-O-GlcNAc-TAB1 and CTD110.6). ${ }^{24,108}$

In future the utility of $\mathrm{CHF}$ or $\mathrm{CF}_{2}$ functionalized GlcNAc as potentially nonhydrolyzable analogues for EPL could be investigated as these have been shown to be good steric and electronic representations in the context of O-phosphorylation. ${ }^{109}$ The main disadvantage of EPL is that it must be optimised for every protein and the maximum length of the peptide for ligation is

a

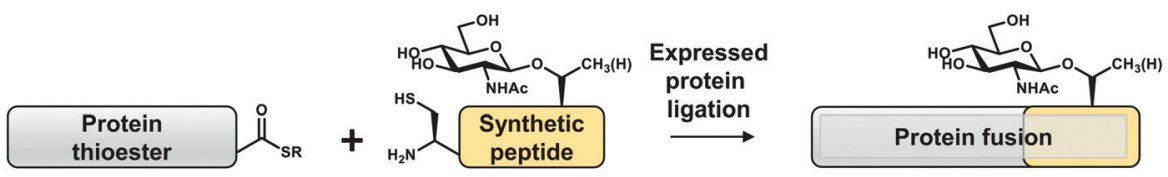

b

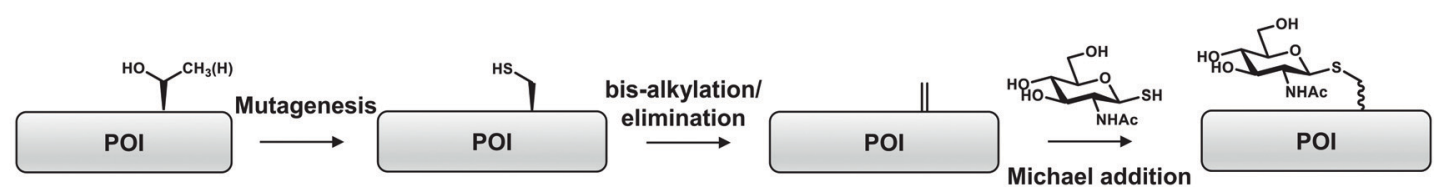

Fig. 3 Site-specific chemical biology approaches to study O-GlcNAcylation in vitro. (a) Expressed protein ligation. (b) "Tag-and-modify" dehydroalanine approach. POI - protein of interest. 
currently limited. Importantly, this method requires good expression of a soluble intein-fusion protein, which frequently poses a problem. Insertion of modified peptides into the central portions of large, globular proteins involves iterative ligation steps and is particularly challenging. A cysteine residue that EPL introduces at the site of ligation cannot always be converted to a native amino acid (e.g. to alanine via desulphurisation). Therefore, the site of ligation must be carefully considered.

In another approach, taking advantage of the potent nucleophilicity and low abundance of cysteine in proteins, cysteineconversion chemical methods have been developed by Davis and co-workers to obtain proteins with site-specific O-GlcNAc mimics in vitro (Fig. 3b). ${ }^{110-112}$ These rely on the chemical transformation of a genetically installed cysteine into dehydroalanine by an alkylating reagent (for example, O-mesitylenesulfonylhydroxylamine (MSH) or 2,5-dibromohexanediamide (DBHDA)) under denaturing conditions. The dehydroalanine of the unfolded protein is then incubated with a reactive GlcNAc derivative (e.g. GlcNAc-thiol) and the modified protein is refolded. ${ }^{110-112}$ Notably, a thiol-linked GlcNAc prepared in such a way is resistant to reduction by DTT. ${ }^{113}$ Moreover, endoglycosidase-A exhibits trans-glycosylation activity for a synthetic s-linked GlcNAc (S-GlcNAc)-modified protein with a high modification efficiency. ${ }^{112}$ Cys-S-GlcNAcylation at position 101 (Thr in the native protein) of histone $\mathrm{H} 2 \mathrm{~A}$ was shown to destabilise $\mathrm{H} 2 \mathrm{~A} / \mathrm{H} 2 \mathrm{~B}$ dimers, promoting an open chromatin state. Installation of S-GlcNAc at position 112 of histone H2B (Ser in the native protein) allowed identification of interactors, among which are subunits of the FACT chromatin remodelling complex. ${ }^{110,111}$ As evidence for physiological mimicry, O-GlcNAc-homohomo-Ser prepared by this approach can be hydrolysed by human OGA. ${ }^{114}$ This method is limited to recombinant proteins with no or few native cysteine residues, that otherwise must first be substituted by other amino acids (Ser or Ala). Furthermore, this approach does not allow control of stereo-specificity of the modification rendering it difficult to interpret the results of the experiment.

Another method for installing S-GlcNAc employs a thioglycoligase capable of transferring GlcNAc to cysteines, engineered from a Streptomyces plicatus hexosaminidase via mutation of the catalytic glutamate (E314A). ${ }^{115}$ Withers and co-workers managed to produce S-GlcNAcylated synuclein peptides and tau protein using this new method. ${ }^{115}$ As with the dehydroalanine approach, this technique requires mutation of all native cysteines that are not destined for S-GlcNAc modification and its utility is limited to in vitro reactions.

\section{Genetic methods for dissecting site-specific O-GlcNAcylation in vivo}

Loss-of-function mutations are the most common approach to probe site-specific O-GlcNAcylation in cultured cells (e.g. Ser/Thr mutation to Ala). For example, Ser529Ala mutation on phosphofructokinase 1 was shown to prevent O-GlcNAcdependent increase in its activity, ${ }^{116}$ while Thr228Ala mutation of Oct4 reduces stem cell self-renewal and reprogramming. ${ }^{117}$ The caveat of this approach lies in the loss of the side chain which in itself could have an impact on protein folding and stability. Therefore, Ala mutagenesis is often used in combination with other methods to down- or upregulate OGT or OGA levels and their enzymatic activity. OGA knockout, knockdown and inhibition is an effective way to elevate total O-GlcNAc modification levels in vivo and in cell culture, since O-GlcNAcylation is often sub-stoichiometric in cells $(<10 \%)$ due to the high OGA activity. ${ }^{118-120}$ However, with this approach, functional dissection of the roles of individual O-GlcNAc sites is impeded by potential ambiguous phenotypes through effects on many other OGT substrates.

Genetic code expansion (GCE) technology allows sitespecific incorporation of unnatural amino acids (including PTMs) in vitro and in vivo by utilising evolved orthogonal amber suppressor tRNA synthetases (Fig. 4a). ${ }^{121-125}$ The GCE method often requires evolution of an archaeal pyrrolysine-tRNA synthetase (PylRS) to aminoacylate a corresponding amber suppressor tRNA, which in turn allows decoding of the unnatural amino acid (UAA) of interest at an amber stop (or quadruplet $^{121}$ ) codon in a genetically predetermined fashion (Fig. 4a). Since the amber codon is the least abundant of the three stop codons, its use reduces the off-target incorporation of the amino acid where endogenous amber stop codons occur.

Co-translational incorporation of a glycosylated amino acid by the GCE technology could become a promising tool to sitespecifically examine the gain-of-function O-GlcNAc modification in vivo. ${ }^{122-124}$ With over 200 UAAs incorporated to date, ${ }^{126}$ the expanded genetic code includes several PTMs such as phosphorylated amino acids (serine, threonine and tyrosine), acetylated lysine and a scaffold for ubiquitin- and SUMOmodified lysine. ${ }^{127-132}$ Most of the UAAs incorporated by the PylRS-based GCE are lysine derivatives that resemble the natural substrate of PylRS, pyrrolysine. ${ }^{126}$ Amino acids as polar as GlcNAcylated serine or cysteine have not yet been added to the expanded genetic code. Apart from the specificity and activity of the evolved PylRS, incorporation of UAAs largely depends on the tolerance of the corresponding aminoacylated tRNA by the translation machinery, such as the ribosome, elongation factor Tu and release factors. These problems were previously encountered with phosphoserine, for which some components of the translational machinery had to be additionally engineered. ${ }^{128}$

A major obstacle in applying GCE to O-GlcNAc is the uptake and stability of the synthetic O-GlcNAcylated amino acid. E. coli, which is used as a host system for evolving PylRS, metabolises Ser-O-GlcNAc as a carbon source, ${ }^{133}$ precluding the use of this UAA for GCE. ${ }^{134}$ De-acetylation of a per-O-acetylated Ser-O-GlcNAc variant (which would hypothetically increase the cellular uptake) does not occur in $E$. coli as it does not express the requisite deacetylases. ${ }^{133}$ The latter strategy would be more suited to mammalian cells, where pan-specific de-acetylating enzymes do exist. Importantly, however, we showed that a synthetic amino acid Cys-S-GlcNAc, a Ser-O-GlcNAc analogue, is efficiently internalised by $E$. coli where it remains metabolically stable, satisfying the first essential step and prerequisite for directed evolution of PylRS. ${ }^{135}$

Recombinant proteins with a glycosylation mimic have been obtained indirectly through PylRS amber suppression, by 
a

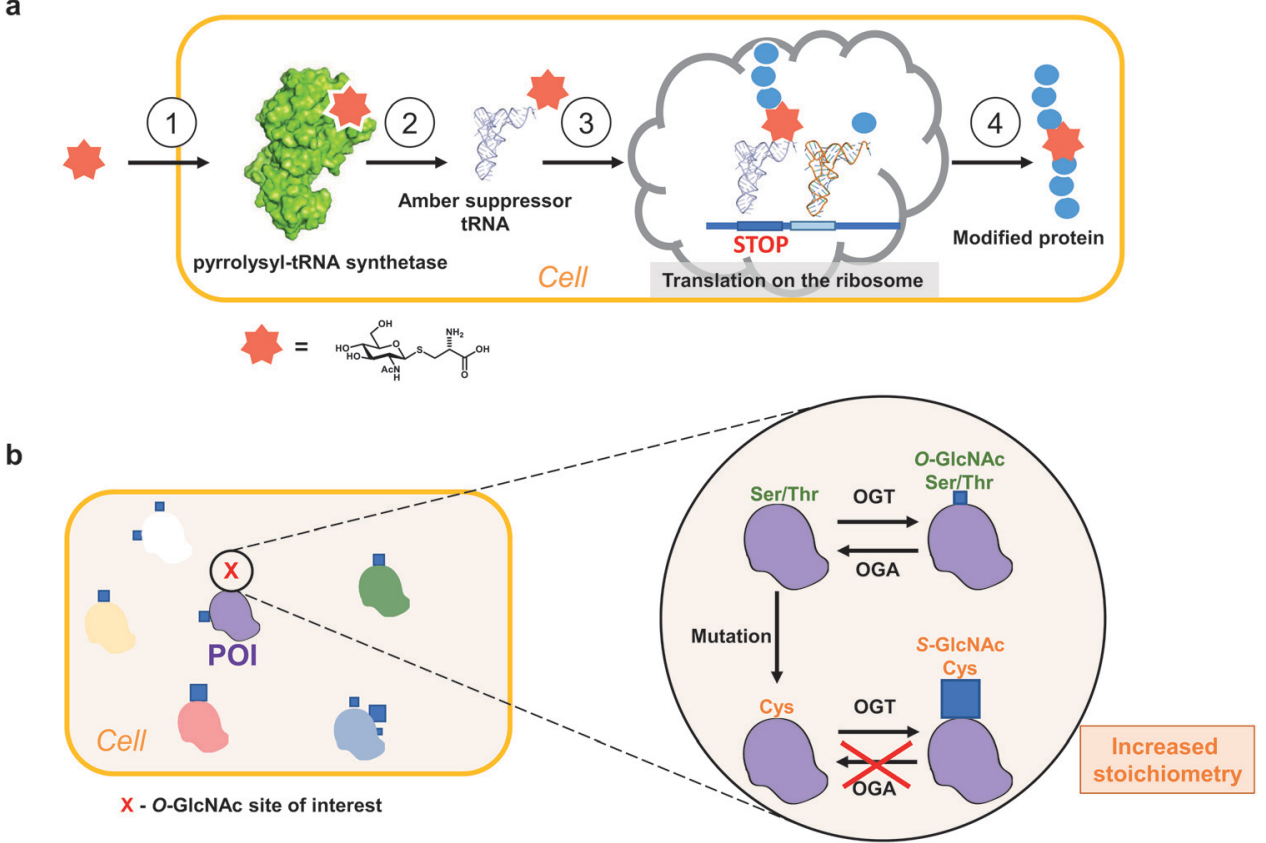

Fig. 4 Approaches to study site-specific O-GlcNAcylation in vivo. (a) Principle of a genetic code expansion approach with a metabolically stable Cys-SGIcNAc amino acid: (1). UAA uptake, (2). tRNA aminoacylation, (3). UAA translation in response to a stop codon, (4). Production of a modified protein. (b) Genetic recoding approach to introduce site-specific S-GlcNAc mimicry of O-GlcNAcylation. ${ }^{24}$ Blue squares denote GlcNAc. POI - protein of interest.

introducing an amino acid with the alkene functionality followed by a click chemistry reaction. ${ }^{136}$ Recently, cellular incorporation of dehydroalanine was demonstrated. ${ }^{137}$ This allowed the authors to install thio-linked GlcNAc at a defined position on GFP which was recognised by a pan-specific O-GlcNAc antibody. ${ }^{137}$

Although glycosylated amino acids have not been incorporated by GCE, there are some clues as to the feasibility of this approach reported in several studies. ${ }^{138,139}$ In these reports glycosylated recombinant proteins were produced in a cell-free system using chemically aminoacylated amber suppressor tRNAs. ${ }^{138,139}$ While the efficiencies of amber suppression varied between different glycosylated amino acids reaching up to $30 \%,{ }^{138}$ in one of the studies, it was shown that Thr-O-GalNAc could be incorporated only at the protein N-terminus, suggesting that glycosylated polypeptides are not well tolerated by the ribosome. ${ }^{139}$

The major advantage of GCE is the ability to produce stoichiometrically-modified proteins in living systems. On the other hand, the level of incorporation strongly depends on the suppression efficiency of the stop codon. This does not pose significant issues for purified recombinant proteins (where the expression can be scaled up). However, when interpreting the effects of a post-translational modification in vivo, it is challenging to provide appropriate controls (i.e. achieving the same expression as for the unmodified protein). Therefore, any observed effects resulting from incorporation of a UAA could also be the result of altered expression levels. Undesired offtarget effects could also be observed in case of mis-incorporation of the amino acid in unintended proteins in place of endogenous stop codons, resulting in skewed phenotypes. Future developments of GCE are required to address these issues.
To gain insight into site-specific O-GlcNAcylation in cells, our laboratory has explored a surprisingly simple approach to genetically introduce a non-hydrolysable S-GlcNAc in vitro and in vivo using Ser/Thr to Cys mutagenesis and relying on the fortuitous promiscuity of OGT that happens to possess efficient S-GlcNAc transferase activity (Fig. $4 \mathrm{~b}$ ). ${ }^{24}$ Notably, this method does not require any exogenous biomolecular machinery, overexpression of (mutant) genes or chemical synthesis and can be combined with the CRISPR-Cas9 genome editing technology. With this approach, the stoichiometry of cysteine GlcNAcylation can replicate that of OGA inhibition in cells due to the hydrolytic stability of S-GlcNAc. However, unlike OGA inhibition, S-GlcNAc mutagenesis does not affect global O-GlcNAc levels, minimizing undesired indirect effects. By applying this method to a single Ser O-GlcNAcylation site on OGA and converting it to a Cys S-GlcNAcylation site, we managed to increase the stoichiometry almost five-fold (from $15 \%$ to over $70 \%$ ), similar to that achieved with the OGA inhibitor treatment. ${ }^{24}$ We showed that the high GlcNAcylation stoichiometry decreased OGA stability in cells, while the overall thermal stability remained unchanged relative to the wild type protein. ${ }^{24}$ Interestingly, in the same sequence contexts S-GlcNAc appears to be more stable to CID MS/MS fragmentation ${ }^{23}$ and may assist in mapping and detection of O-GlcNAc sites. Additionally, homogenous site-specifically S-GlcNAcylated recombinant proteins can be produced through GlcNAcylation of a Cys mutant protein and subsequent treatment with OGA (for example, the highly-active $C p \mathrm{OGA}$ ) to remove unwanted O-GlcNAcylation (if multiple modification sites are present). It must be noted that in cases where OGA inhibition does not elevate O-GlcNAcylation levels on a protein of interest in cells, this approach may not be applicable. While this 
S-GlcNAc genetic recoding approach has been applied in cultured human and mouse cells, it has yet to be demonstrated that this leads to site-specific S-GlcNAc incorporation in animal models.

\section{Conclusions}

The functional consequences of protein O-GlcNAcylation still remain poorly understood due to the limited number of tools to study its site-specific effects. As can be appreciated from this review, no single method to study protein- and site-specific O-GlcNAcylation can be universally applied and all of the discussed techniques have their advantages and disadvantages. Thus, a combination of these approaches can help us achieve a comprehensive understanding of the functions of the O-GlcNAc PTM on specific proteins and sites. The future developments in the field require optimization of contemporary methods and invention of new strategies to gain control of protein- and site-specific modification. Expanding the genetic code with glycosylated amino acids would represent just one of these future advances. Ingenious methods are required to achieve protein- and site-specific O-GlcNAcylation with minimal side effects and to avoid unnecessary non-physiological perturbation in living systems. The use of non-hydrolysable analogues of O-GlcNAc (such as S-, CHF- and $\mathrm{CF}_{2}$-linked GlcNAc or other analogues ${ }^{24,74,140}$ ) in an intracellular setting must be considered to withstand the high activity of OGA that could otherwise abolish the efforts of site-specific installation of O-GlcNAc. Spatiotemporal control of site-specific O-GlcNAcylation in cells is also important, since O-GlcNAc is a signalling molecule that can lead to rapid activation of signalling cascades. Last but not least, low O-GlcNAcylation stoichiometry at a given site can be the result of protein-protein interactions that "mask" such modification sites and prevent subsequent posttranslational modification by OGT, thus requiring implementation of techniques for co-translational site-specific GlcNAc incorporation. Bridging the gap between chemistry and biology is required to invent approaches that fine-tune O-GlcNAc stoichiometry with a single protein and amino acid residue precision. Such interdisciplinary amalgamation will be instrumental in investigating the diverse functions of protein O-GlcNAcylation.

\section{Conflicts of interest}

There are no conflicts of interest to declare.

\section{Acknowledgements}

This work was funded by a Wellcome Trust Investigator Award (110061) to DvA and a Wellcome Trust 4-year PhD studentship $(105310 / \mathrm{Z} / 14 / \mathrm{Z})$ to AG. Fig. 2a and b were created with biorender.com.

\section{References}

1 R. Aebersold, et al., How many human proteoforms are there?, Nat. Chem. Biol., 2018, 14, 206-214.
2 T. Klein, U. Eckhard, A. Dufour, N. Solis and C. M. Overall, Proteolytic Cleavage - Mechanisms, Function, and 'omic' Approaches for a Near-Ubiquitous Posttranslational Modification, Chem. Rev., 2018, 118, 1137-1168.

3 R. Yau and M. Rape, The increasing complexity of the ubiquitin code, Nat. Cell Biol., 2016, 18, 579-586.

4 K. W. Moremen, M. Tiemeyer and A. V. Nairn, Vertebrate protein glycosylation: Diversity, synthesis and function, Nat. Rev. Mol. Cell Biol., 2012, 13, 448-462.

5 F. Ardito, M. Giuliani, D. Perrone, G. Troiano and L. L. Muzio, The crucial role of protein phosphorylation in cell signaling and its use as targeted therapy (Review), Int. J. Mol. Med., 2017, 40, 271-280.

6 E. Verdin and M. Ott, 50 years of protein acetylation: From gene regulation to epigenetics, metabolism and beyond, Nat. Rev. Mol. Cell Biol., 2015, 16, 258-264.

7 A. Jambhekar, A. Dhall and Y. Shi, Roles and regulation of histone methylation in animal development, Nat. Rev. Mol. Cell Biol., 2019, 20, 625-641.

8 B. Chen, Y. Sun, J. Niu, G. K. Jarugumilli and X. Wu, Protein Lipidation in Cell Signaling and Diseases: Function, Regulation, and Therapeutic Opportunities, Cell Chem. Biol., 2018, 25, 817-831.

9 G. W. Hart, Nutrient regulation of signaling and transcription, J. Biol. Chem., 2019, 294, 2211-2231.

10 Y. Zhu, et al., O-GlcNAc occurs cotranslationally to stabilize nascent polypeptide chains, Nat. Chem. Biol., 2015, 11, 319-325.

11 R. S. Haltiwanger, M. A. Blomberg and G. W. Hart, Glycosylation of nuclear and cytoplasmic proteins: Purification and characterization of a uridine diphospho- $\mathrm{N}$-acetylglucosamine: polypeptide beta- $N$-acetylglucosaminyltransferase, J. Biol. Chem., 1992, 267, 9005-9013.

12 S. Pathak, et al., The active site of O-GlcNAc transferase imposes constraints on substrate sequence, Nat. Struct. Mol. Biol., 2015, 22, 744-750.

13 J. Ma and G. W. Hart, Protein O-GlcNAcylation in diabetes and diabetic complications, Expert Rev. Proteomics, 2013, 10, 365-380.

14 Y. Gao, L. Wells, F. I. Comer, G. J. Parker and G. W. Hart, Dynamic O-glycosylation of nuclear and cytosolic proteins: Cloning and characterization of a neutral, cytosolic beta- $N$ acetylglucosaminidase from human brain, J. Biol. Chem., 2001, 276, 9838-9845.

15 X. Yang and K. Qian, Protein O-GlcNAcylation: emerging mechanisms and functions, Nat. Rev. Mol. Cell Biol., 2017, 18, 452-465.

16 B. I. Cantarel, et al., The Carbohydrate-Active EnZymes database (CAZy): An expert resource for glycogenomics, Nucleic Acids Res., 2009, 37, D233-D238.

17 M. B. Lazarus, Y. Nam, J. Jiang, P. Sliz and S. Walker, Structure of human O-GlcNAc transferase and its complex with a peptide substrate, Nature, 2011, 469, 564-567.

18 D. Nolte and U. Müller, Human O-GlcNAc transferase (OGT): Genomic structure, analysis of splice variants, fine mapping in Xq13.1, Mamm. Genome, 2002, 13, 62-64. 
19 J. A. Hanover, et al., Mitochondrial and nucleocytoplasmic isoforms of O-linked GlcNAc transferase encoded by a single mammalian gene, Arch. Biochem. Biophys., 2003, 409, 287-297.

20 R. Trapannone, D. Mariappa, A. T. Ferenbach and D. M. F. van Aalten, Nucleocytoplasmic human O-GlcNAc transferase is sufficient for O-GlcNAcylation of mitochondrial proteins, Biochem. J., 2016, 473, 1693-1702.

21 M. Schimpl, et al., O-GlcNAc transferase invokes nucleotide sugar pyrophosphate participation in catalysis, Nat. Chem. Biol., 2012, 8, 969-974.

22 M. B. Lazarus, et al., HCF-1 is cleaved in the active site of O-GlcNAc transferase, Science, 2013, 342, 1235-1239.

23 J. C. Maynard, A. L. Burlingame and K. F. Medzihradszky, Cysteine S-linked $N$-acetylglucosamine (S-GlcNAcylation), A New Post-translational Modification in Mammals, Mol. Cell. Proteomics, 2016, 15, 3405-3411.

24 A. Gorelik, et al., Genetic recoding to dissect the roles of site-specific protein O-GlcNAcylation, Nat. Struct. Mol. Biol., 2019, 26, 1071-1077.

25 M. Jínek, et al., The superhelical TPR-repeat domain of O-linked GlcNAc transferase exhibits structural similarities to importin $\alpha$, Nat. Struct. Mol. Biol., 2004, 11, 1001-1007.

26 K. Rafie, et al., Recognition of a glycosylation substrate by the O-GlcNAc transferase TPR repeats, Open Biol., 2017, 7, 170078.

27 Z. G. Levine, et al., O-GlcNAc Transferase Recognizes Protein Substrates Using an Asparagine Ladder in the Tetratricopeptide Repeat (TPR) Superhelix, J. Am. Chem. Soc., 2018, 140, 3510-3513.

28 C. M. Joiner, Z. G. Levine, C. Aonbangkhen, C. M. Woo and S. Walker, Aspartate Residues Far from the Active Site Drive O-GlcNAc Transferase Substrate Selection, J. Am. Chem. Soc., 2019, 141, 12974-12978.

29 J. F. Alfaro, et al., Tandem mass spectrometry identifies many mouse brain O-GlcNAcylated proteins including EGF domain-specific O-GlcNAc transferase targets, Proc. Natl. Acad. Sci. U. S. A., 2012, 109, 7280-7285.

30 Z. Wang, et al., Enrichment and site mapping of O-linked $\mathrm{N}$-acetylglucosamine by a combination of chemical/enzymatic tagging, photochemical cleavage, and electron transfer dissociation mass spectrometry, Mol. Cell. Proteomics, 2010, 9, 153-160.

31 R. J. Chalkley, A. Thalhammer, R. Schoepfer and A. L. Burlingame, Identification of protein O-GlcNAcylation sites using electron transfer dissociation mass spectrometry on native peptides, Proc. Natl. Acad. Sci. U. S. A., 2009, 106, 8894-8899.

32 F. V. Rao, et al., Structure of a bacterial putative acetyltransferase defines the fold of the human O-GlcNAcase C-terminal domain, Open Biol., 2013, 3, 130021.

33 N. L. Elsen, et al., Insights into activity and inhibition from the crystal structure of human O-GlcNAcase, Nat. Chem. Biol., 2017, 13, 613-615.

34 C. N. Keembiyehetty, A. Krzeslak, D. C. Love and J. A. Hanover, A lipid-droplet-targeted O-GlcNAcase isoform is a key regulator of the proteasome, J. Cell Sci., 2011, 124, 2851-2860.

35 F. V. Rao, et al., Structural insights into the mechanism and inhibition of eukaryotic O-GlcNAc hydrolysis, EMBO J., 2006, 25, 1569-1578.

36 M. Schimpl, V. S. Borodkin, L. J. Gray and D. M. van Aalten, Synergy of peptide and sugar in O-GlcNAcase substrate recognition, Chem. Biol., 2012, 19, 173-178.

37 B. Li, H. Li, L. Lu and J. Jiang, Structures of human O-GlcNAcase and its complexes reveal a new substrate recognition mode, Nat. Struct. Mol. Biol., 2017, 24, 362-369.

38 C. Roth, et al., Structural and functional insight into human O-GlcNAcase, Nat. Chem. Biol., 2017, 13, 610-612.

39 B. Li, H. Li, C. W. Hu and J. Jiang, Structural insights into the substrate binding adaptability and specificity of human O-GlcNAcase, Nat. Commun., 2017, 8, 666.

$40 \mathrm{H}$. G. Seo, et al., Identification of the nuclear localisation signal of O-GlcNAc transferase and its nuclear import regulation, Sci. Rep., 2016, 6, 34614.

41 R. Shafi, et al., The O-GlcNAc transferase gene resides on the $\mathrm{X}$ chromosome and is essential for embryonic stem cell viability and mouse ontogeny, Proc. Natl. Acad. Sci. U. S. A., 2000, 97, 5735-5739.

42 M. C. Gambetta, K. Oktaba and J. Muller, Essential role of the glycosyltransferase sxc/Ogt in polycomb repression, Science, 2009, 325, 93-96.

43 D. Mariappa, A. T. Ferenbach and D. M. F. Van Aalten, Effects of hypo O-GlcNAcylation on Drosophila development, J. Biol. Chem., 2018, 293, 7209-7221.

44 Y. R. Yang, et al., O-GlcNAcase is essential for embryonic development and maintenance of genomic stability, Aging Cell, 2012, 11, 439-448.

45 C. Slawson and G. W. Hart, O-GlcNAc signalling: implications for cancer cell biology, Nat. Rev. Cancer, 2011, 11, 678-684.

46 G. W. Hart, C. Slawson, G. Ramirez-Correa and O. Lagerlof, Cross talk between O-GlcNAcylation and phosphorylation: roles in signaling, transcription, and chronic disease, Annu. Rev. Biochem., 2011, 80, 825-858.

47 M. R. Martinez, T. B. Dias, P. S. Natov and N. E. Zachara, Stress-induced O-GlcNAcylation: An adaptive process of injured cells, Biochem. Soc. Trans., 2017, 45, 237-249.

48 F. Liu, K. Iqbal, I. Grundke-Iqbal, G. W. Hart and C.-X. Gong, O-GlcNAcylation regulates phosphorylation of tau: A mechanism involved in Alzheimer's disease, Proc. Natl. Acad. Sci. U. S. A., 2004, 101, 10804-10809.

49 N. P. Marotta, et al., O-GlcNAc modification blocks the aggregation and toxicity of the protein alpha-synuclein associated with Parkinson's disease, Nat. Chem., 2015, 7, 913-920.

50 S. M. Ranuncolo, S. Ghosh, J. A. Hanover, G. W. Hart and B. A. Lewis, Evidence of the involvement of O-GlcNAcmodified human RNA polymerase II CTD in transcription in vitro and in vivo, J. Biol. Chem., 2012, 287, 23549-23561.

51 B. Guo, et al., O-GlcNAc-modification of SNAP-29 regulates autophagosome maturation, Nat. Cell Biol., 2014, 16, 1215-1226. 
52 S. K. Park, et al., A Conserved Splicing Silencer Dynamically Regulates O-GlcNAc Transferase Intron Retention and O-GlcNAc Homeostasis, Cell Rep., 2017, 20, 1088-1099.

53 Z. Zhang, E. P. Tan, N. J. VandenHull, K. R. Peterson and C. Slawson, O-GlcNAcase Expression is Sensitive to Changes in O-GlcNAc Homeostasis, Front. Endocrinol., 2014, 5, 206.

$54 \mathrm{~K}$. Vaidyanathan, et al., Identification and characterization of a missense mutation in the O-linked $\beta$ - $N$-acetylglucosamine (O-GlcNAc) transferase gene that segregates with X-linked intellectual disability, J. Biol. Chem., 2017, 292, 8948-8963.

55 A. P. Willems, et al., Mutations in $N$-acetylglucosamine (O-GlcNAc) transferase in patients with X-linked intellectual disability, J. Biol. Chem., 2017, 292, 12621-12631.

56 N. Selvan, et al., O-GlcNAc transferase missense mutations linked to X-linked intellectual disability deregulate genes involved in cell fate determination and signaling, J. Biol. Chem., 2018, 293, 10810-10824.

57 V. M. Pravata, et al., A missense mutation in the catalytic domain of O-GlcNAc transferase links perturbations in protein O-GlcNAcylation to X-linked intellectual disability, FEBS Lett., 2019, 594, 717-727.

58 V. M. Pravata, et al., Catalytic deficiency of O-GlcNAc transferase leads to X-linked intellectual disability, Proc. Natl. Acad. Sci. U. S. A., 2019, 116, 14961-14970.

59 M. C. Gambetta and J. Müller, O-GlcNAcylation Prevents Aggregation of the Polycomb Group Repressor Polyhomeotic, Dev. Cell, 2014, 31, 629-639.

$60 \mathrm{X}$. Li, et al., O-GlcNAc Transferase Suppresses Inflammation and Necroptosis by Targeting Receptor-Interacting Serine/ Threonine-Protein Kinase 3, Immunity, 2019, 50, 576-590.

$61 \mathrm{H}$. Nie, et al., O-GlcNAcylation of PGK1 coordinates glycolysis and TCA cycle to promote tumor growth, Nat. Commun., 2020, 11, 36.

$62 \mathrm{~J}$. W. Thompson, A. W. Sorum and L. C. Hsieh-Wilson, Deciphering the Functions of O-GlcNAc Glycosylation in the Brain: The Role of Site-Specific Quantitative O-GlcNAcomics, Biochemistry, 2018, 57, 4010-4018.

63 W. G. Kelly and G. W. Hart, Glycosylation of chromosomal proteins: Localization of O-linked $\mathrm{N}$-acetylglucosamine in Drosophila chromatin, Cell, 1989, 57, 243-251.

64 G. D. Holt, et al., Nuclear pore complex glycoproteins contain cytoplasmically disposed O-linked $N$-acetylglucosamine, J. Cell Biol., 1987, 104, 1157-1164.

65 N. Khidekel, et al., A chemoenzymatic approach toward the rapid and sensitive detection of O-GlcNAc posttranslational modifications, J. Am. Chem. Soc., 2003, 125, 16162-16163.

66 J. E. Rexach, et al., Quantification of O-glycosylation stoichiometry and dynamics using resolvable mass tags, Nat. Chem. Biol., 2010, 6, 645-651.

67 C. F. Teo and L. Wells, Monitoring protein O-linked $\beta-N$ acetylglucosamine status via metabolic labeling and copperfree click chemistry, Anal. Biochem., 2014, 464, 70-72.

68 P. M. Clark, et al., Direct in-gel fluorescence detection and cellular imaging of O-GlcNAc-modified proteins, J. Am. Chem. Soc., 2008, 130, 11576-11577.
69 N. Darabedian, J. W. Thompson, K. N. Chuh, L. C. HsiehWilson and M. R. Pratt, Optimization of Chemoenzymatic Mass Tagging by Strain-Promoted Cycloaddition (SPAAC) for the Determination of O-GlcNAc Stoichiometry by Western Blotting, Biochemistry, 2018, 57, 5769-5774.

70 P. V. Robinson, C. T. Tsai, A. E. De Groot, J. L. McKechnie and C. R. Bertozzi, Glyco-seek: Ultrasensitive Detection of Protein-Specific Glycosylation by Proximity Ligation Polymerase Chain Reaction, J. Am. Chem. Soc., 2016, 138, 10722-10725.

71 D. J. Vocadlo, H. C. Hang, E.-J. Kim, J. A. Hanover and C. R. Bertozzi, A chemical approach for identifying O-GlcNAc-modified proteins in cells, Proc. Natl. Acad. Sci. U. S. A., 2003, 100, 9116-9121.

72 B. W. Zaro, Y.-Y. Yang, H. C. Hang and M. R. Pratt, Chemical reporters for fluorescent detection and identification of O-GlcNAc-modified proteins reveal glycosylation of the ubiquitin ligase NEDD4-1, Proc. Natl. Acad. Sci. U. S. A., 2011, 108, 8146-8151.

73 K. N. Chuh, B. W. Zaro, F. Piller, V. Piller and M. R. Pratt, Changes in metabolic chemical reporter structure yield a selective probe of O-GlcNAc modification, J. Am. Chem. Soc., 2014, 136, 12283-12295.

$74 \mathrm{~J}$. Li, et al., An OGA-resistant probe allows specific visualization and accurate identification of O-GlcNAc-modified proteins in cells, ACS Chem. Biol., 2016, 11, 3002-3006.

75 B. W. Zaro, A. R. Batt, K. N. Chuh, M. X. Navarro and M. R. Pratt, The Small Molecule 2-Azido-2-deoxy-glucose Is a Metabolic Chemical Reporter of O-GlcNAc Modifications in Mammalian Cells, Revealing an Unexpected Promiscuity of O-GlcNAc Transferase, ACS Chem. Biol., 2017, 12, 787-794.

76 K. N. Chuh, et al., The New Chemical Reporter 6-Alkynyl-6deoxy-GlcNAc Reveals O-GlcNAc Modification of the Apoptotic Caspases That Can Block the Cleavage/Activation of Caspase-8, J. Am. Chem. Soc., 2017, 139, 7872-7885.

77 N. Darabedian, J. Gao, K. N. Chuh, C. M. Woo and M. R. Pratt, The metabolic chemical reporter 6-azido-6deoxy-glucose further reveals the substrate promiscuity of O-GlcNAc transferase and catalyzes the discovery of intracellular protein modification by O-glucose, J. Am. Chem. Soc., 2017, 140, 7092-7100.

78 S.-H. Yu, et al., Metabolic labeling enables selective photocrosslinking of O-GlcNAc-modified proteins to their binding partners, Proc. Natl. Acad. Sci. U. S. A., 2012, 109, 4834-4839.

79 C. M. Snow, A. Senior and L. Gerace, Monoclonal antibodies identify a group of nuclear pore complex glycoproteins, J. Cell Biol., 1987, 104, 1143-1156.

80 F. I. Comer, K. Vosseller, L. Wells, M. A. Accavitti and G. W. Hart, Characterization of a mouse monoclonal antibody specific for O-linked $\mathrm{N}$-acetylglucosamine, Anal. Biochem., 2001, 293, 169-177.

81 T. Isono, O-GlcNAc-specific antibody CTD110.6 crossreacts with N-GlcNAc2-modified proteins induced under glucose deprivation, PLoS One, 2011, 6, e18959.

82 M. Ogawa, et al., GTDC2 modifies O-mannosylated $\alpha$-dystroglycan in the endoplasmic reticulum to generate 
$\mathrm{N}$-acetyl glucosamine epitopes reactive with CTD110.6 antibody, Biochem. Biophys. Res. Commun., 2013, 440, 88-93.

83 Y. Tashima and P. Stanley, Antibodies that detect O-linked beta-D- $N$-acetylglucosamine on the extracellular domain of cell surface glycoproteins, J. Biol. Chem., 2014, 289, 11132-11142.

84 R. A. Reeves, A. Lee, R. Henry and N. E. Zachara, Characterization of the specificity of O-GlcNAc reactive antibodies under conditions of starvation and stress, Anal. Biochem., 2014, 457, 8-18.

85 P. M. Levine, et al., $\alpha$-Synuclein O-GlcNAcylation alters aggregation and toxicity, revealing certain residues as potential inhibitors of Parkinson's disease, Proc. Natl. Acad. Sci. U. S. A., 2019, 116, 1511-1519.

86 K. Kamemura, B. K. Hayes, F. I. Comer and G. W. Hart, Dynamic interplay between O-glycosylation and O-phosphorylation of nucleocytoplasmic proteins: Alternative glycosylation/phosphorylation of Thr-58, a known mutational hot spot of c-Myc in lymphomas, is regulated by mitogens, J. Biol. Chem., 2002, 277, 19229-19235.

87 S. Pathak, et al., O-GlcNAcylation of TAB1 modulates TAK1mediated cytokine release, EMBO J., 2012, 31, 1394-1404.

88 V. Muha, et al., Loss of CRMP2 O-GlcNAcylation leads to reduced novel object recognition performance in mice, Open Biol., 2019, 9, 190192.

89 M. Hirosawa, et al., Novel O-GlcNAcylation on Ser40 of canonical H2A isoforms specific to viviparity, Sci. Rep., 2016, 6, 31785.

90 R. Fujiki, et al., GlcNAcylation of histone H2B facilitates its monoubiquitination, Nature, 2011, 480, 557-560.

91 A. L. Klein, M. N. Berkaw, M. G. Buse and L. E. Ball, O-linked $\mathrm{N}$-acetylglucosamine modification of insulin receptor substrate-1 occurs in close proximity to multiple $\mathrm{SH} 2$ domain binding motifs, Mol. Cell. Proteomics, 2009, 8, 2733-2745.

92 H. Shan, J. Sun, M. Shi, X. Liu, Z. Shi, W. Yu and G. Yuchao, Generation and characterization of a site-specific antibody for SIRT1 O-GlcNAcylated at serine 549, Glycobiology, 2018, 28, 482-487.

93 S. A. Yuzwa, et al., Mapping O-GlcNAc modification sites on tau and generation of a site-specific O-GlcNAc tau antibody, Amino Acids, 2011, 40, 857-868.

94 A. Cameron, et al., Generation and characterization of a rabbit monoclonal antibody site-specific for tau O-GlcNAcylated at serine 400, FEBS Lett., 2013, 587, 3722-3728.

95 T. Tao, Z. He, Z. Shao and H. Lu, TAB3 O-GlcNAcylation promotes metastasis of triple negative breast cancer, Oncotarget, 2016, 7, 22807-22818.

96 D. L. Shen, T. M. Gloster, S. A. Yuzwa and D. J. Vocadlo, Insights into O-linked $\mathrm{N}$-acetylglucosamine (O-GlcNAc) processing and dynamics through kinetic analysis of O-GlcNAc transferase and O-GlcNAcase activity on protein substrates, J. Biol. Chem., 2012, 287, 15395-15408.

97 K. H. Lim, C. H. Ha and H. I. Chang, Production of O-GlcNAc modified recombinant proteins in Escherichia coli, J. Microbiol. Biotechnol., 2002, 12, 306-311.

98 K. C. Sohn and S. I. Do, Transcriptional regulation and O-GlcNAcylation activity of zebrafish OGT during embryogenesis, Biochem. Biophys. Res. Commun., 2005, 337, 256-263.

99 O. Y. Goodwin, M. S. Thomasson, A. J. Lin, M. M. Sweeney and M. A. E. Macnaughtan, E. coli sabotages the in vivo production of O-linked $\beta$ - $N$-acetylglucosamine-modified proteins, J. Biotechnol., 2013, 168, 315-323.

100 D. H. Ramirez, et al., Engineering a Proximity-Directed O-GlcNAc Transferase for Selective Protein O-GlcNAcylation in Cells, ACS Chem. Biol., 2020, 15, 1059-1066.

101 S. Yamazoe, et al., Heterobifunctional Molecules Induce Dephosphorylation of Kinases-A Proof of Concept Study, J. Med. Chem., 2019, 63, 2807-2813.

102 T. W. Muir, D. Sondhi and P. A. Cole, Expressed protein ligation: a general method for protein engineering, Proc. Natl. Acad. Sci. U. S. A., 1998, 95, 6705-6710.

103 R. E. Thompson and T. W. Muir, Chemoenzymatic Semisynthesis of Proteins, Chem. Rev., 2020, 120, 3051-3126.

104 S. Schwagerus, O. Reimann, C. Despres, C. Smet-Nocca and C. P. Hackenberger, Semi-synthesis of a tag-free O-GlcNAcylated tau protein by sequential chemoselective ligation, J. Pept. Sci., 2016, 22, 327-333.

105 Y. E. Lewis, et al., O-GlcNAcylation of $\alpha$-Synuclein at Serine 87 Reduces Aggregation without Affecting Membrane Binding, ACS Chem. Biol., 2017, 12, 1020-1027.

106 A. T. Balana, et al., O-GlcNAcylation of small heat shock proteins enhances their anti-amyloid chaperone activity, bioRxiv, 2019, 869909, DOI: 10.1101/869909.

107 M. K. Tarrant, et al., Regulation of CK2 by phosphorylation and O-GlcNAcylation revealed by semisynthesis, Nat. Chem. Biol., 2012, 8, 262-269.

108 C. A. De Leon, P. M. Levine, T. W. Craven and M. R. Pratt, The Sulfur-Linked Analogue of O-GlcNAc (S-GlcNAc) Is an Enzymatically Stable and Reasonable Structural Surrogate for O-GlcNAc at the Peptide and Protein Levels, Biochemistry, 2017, 56, 3507-3517.

109 V. D. Romanenko and V. P. Kukhar, Fluorinated phosphonates: Synthesis and biomedical application, Chem. Rev., 2006, 106, 3868-3935.

110 L. Lercher, et al., Generation of a synthetic GlcNAcylated nucleosome reveals regulation of stability by H2A-Thr101 GlcNAcylation, Nat. Commun., 2015, 6, 7978.

111 R. Raj, L. Lercher, S. Mohammed and B. G. Davis, Synthetic Nucleosomes Reveal that GlcNAcylation Modulates Direct Interaction with the FACT Complex, Angew. Chem., Int. Ed., 2016, 55, 8918-8922.

112 M. Fernández-González, et al., Site-selective chemoenzymatic construction of synthetic glycoproteins using endoglycosidases, Chem. Sci., 2010, 1, 709-715.

113 G. J. L. Bernardes, J. M. Chalker, J. C. Errey and B. G. Davis, Facile conversion of cysteine and alkyl cysteines to dehydroalanine on protein surfaces: Versatile and switchable access to functionalized proteins, J. Am. Chem. Soc., 2008, 130, 5052-5053.

114 T. H. Wright, et al., Posttranslational mutagenesis: A chemical strategy for exploring protein side-chain diversity, Science, 2016, 354, aag1465. 
115 G. Tegl, et al., Facile Formation of $\beta$-thioGlcNAc Linkages to Thiol-Containing Sugars, Peptides, and Proteins using a Mutant GH20 Hexosaminidase, Angew. Chem., Int. Ed., 2019, 58, 1632-1637.

$116 \mathrm{~W}$. Yi, et al., Phosphofructokinase 1 glycosylation regulates cell growth and metabolism, Science, 2012, 337, 975-980.

117 H. Jang, et al., O-GlcNAc regulates pluripotency and reprogramming by directly acting on core components of the pluripotency network, Cell Stem Cell, 2012, 11, 62-74.

118 N. Khidekel, et al., Probing the dynamics of O-GlcNAc glycosylation in the brain using quantitative proteomics, Nat. Chem. Biol., 2007, 3, 339-348.

119 H. C. Dorfmueller, V. S. Borodkin, M. Schimpl and D. M. F. van Aalten, GlcNAcstatins are nanomolar inhibitors of human O-GlcNAcase inducing cellular hyper-OGlcNAcylation, Biochem. J., 2009, 420, 221-227.

120 T. M. Gloster, et al., Hijacking a biosynthetic pathway yields a glycosyltransferase inhibitor within cells, Nat. Chem. Biol., 2011, 7, 174-181.

$121 \mathrm{~J}$. W. Chin, Expanding and reprogramming the genetic code, Nature, 2017, 550, 53-60.

122 R. J. Ernst, et al., Genetic code expansion in the mouse brain, Nat. Chem. Biol., 2016, 12, 776-778.

123 A. Bianco, F. M. Townsley, S. Greiss, K. Lang and J. W. Chin, Expanding the genetic code of Drosophila melanogaster, Nat. Chem. Biol., 2012, 8, 748-750.

124 S. Greiss and J. W. Chin, Expanding the genetic code of an animal, J. Am. Chem. Soc., 2011, 133, 14196-14199.

125 K. Sakamoto, et al., Site-specific incorporation of an unnatural amino acid into proteins in mammalian cells, Nucleic Acids Res., 2002, 30, 4692-4699.

126 A. Dumas, L. Lercher, C. D. Spicer and B. G. Davis, Designing logical codon reassignment - Expanding the chemistry in biology, Chem. Sci., 2015, 6, 50-69.

127 H. Neumann, S. Y. Peak-Chew and J. W. Chin, Genetically encoding $\mathrm{N} \varepsilon$-acetyllysine in recombinant proteins, Nat. Chem. Biol., 2008, 4, 232-234.
128 D. T. Rogerson, et al., Efficient genetic encoding of phosphoserine and its nonhydrolyzable analog, Nat. Chem. Biol., 2015, 11, 496-503.

129 S. Virdee, et al., Traceless and site-specific ubiquitination of recombinant proteins, J. Am. Chem. Soc., 2011, 133, 10708-10711.

130 M. Fottner, et al., Site-specific ubiquitylation and SUMOylation using genetic-code expansion and sortase, Nat. Chem. Biol., 2019, 15, 276-284.

131 M. S. Zhang, et al., Biosynthesis and genetic encoding of phosphothreonine through parallel selection and deep sequencing, Nat. Methods, 2017, 14, 729-736.

132 X. Luo, et al., Genetically encoding phosphotyrosine and its nonhydrolyzable analog in bacteria, Nat. Chem. Biol., 2017, 13, 845-849.

133 A. K. Antonczak, Z. Simova and E. M. Tippmann, A critical examination of Escherichia coli esterase activity, J. Biol. Chem., 2009, 284, 28795-28800.

134 Z. Zhang, et al., Retraction, Science, 2009, 326, 1187.

135 A. Gorelik, Genetic encoding of a stable O-GlcNAc analogue, PhD thesis, University of Dundee, 2018.

136 E. Kaya, et al., Synthesis of threefold glycosylated proteins using click chemistry and genetically encoded unnatural amino acids, ChemBioChem, 2009, 10, 2858-2861.

137 B. Yang, et al., Genetically Introducing Biochemically Reactive Amino Acids Dehydroalanine and Dehydrobutyrine in Proteins, J. Am. Chem. Soc., 2019, 141, 7698-7703.

138 N. E. Fahmi, L. Dedkova, B. Wang, S. Golovine and S. M. Hecht, Site-specific incorporation of glycosylated serine and tyrosine derivatives into proteins, J. Am. Chem. Soc., 2007, 129, 3586-3597.

139 T. Matsubara, K. Iijima, T. Watanabe, T. Hohsaka and T. Sato, Incorporation of glycosylated amino acid into protein by an in vitro translation system, Bioorg. Med. Chem. Lett., 2013, 23, 5634-5636.

$140 \mathrm{H}$. Wang, et al., $\mathrm{Ac}_{4} \mathrm{GlcNAcF}_{3}$, an OGT-tolerated but OGAresistant regulator for O-GlcNAcylation, Bioorg. Med. Chem. Lett., 2019, 29, 802-805. 